\title{
5 RÄUMLICHES UNTERNEHMENSENGAGEMENT (Corporate SPatial Responsibility) UND Ortsführung (Place Leadership) in KLEINSTÄDTEN. \\ Perspektiven für eine neue Ortsführung? EINE ZUSAMMENFÜHRUNG VON ZWEI DISKURSEN
}

\author{
Hans-Hermann ALBERS, Institut für Stadt- und Regionalplanung, \\ Technische Universität Berlin \\ Lech SUWALA, \\ Institut für Stadt- und Regionalplanung, \\ Technische Universität Berlin
}

\section{INHALT}

5.1 Einleitung. 64

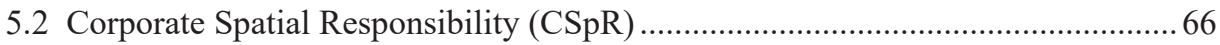

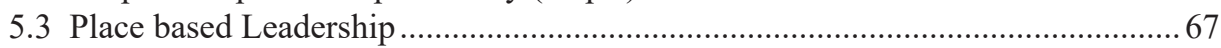

5.4 Zusammenführung von CSpR und Place Leadership ........................................69

5.5 Unternehmerische Engagement- und Führungslandschaften in Kleinstädten ........71

5.6 Diskurs und Einordnung der Initiativen in das Reifegradmodell ..........................75

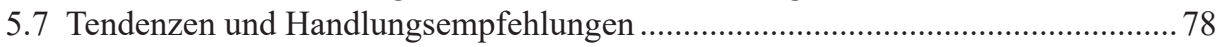

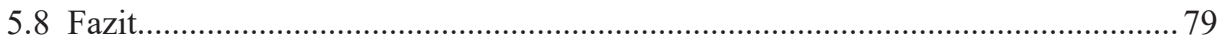

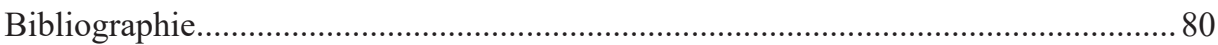

Viele Unternehmen haben ihre gesellschaftliche Verantwortung (Corporate Social Responsibility - CSR) sowohl professionalisiert als auch um die räumliche Dimension erweitert und nehmen - gerade in kleinen Städten und im ländlichen Raum - eine aktive Rolle in der Stadt- und Regionalentwicklung ein. Dieses Handeln lässt sich 
unter dem Begriff Corporate Spatial Responsibility (CSpR) zusammenfassen. Damit die meist partnerschaftlich organisierten Vorhaben gelingen, ist die funktionierende Zusammenarbeit der Akteure und Institutionen unentbehrlich. Je nach Intensität des Engagements kann es sogar zur Etablierung sogenannter, alternativer ortsspezifischer Organisations- und Führungsstrukturen kommen (Place Leadership). Diese wirken dann bestenfalls abgestimmt und ergänzend zu den formellen Verwaltungsstrukturen. Der Beitrag stellt die CSpR - und Place Leadership Diskurse vor, formuliert eine Kombination der beiden Konzepte und diskutiert die Einordnung anhand von Fallbeispielen.

\subsection{Einleitung}

Unternehmen übernehmen zunehmend gesellschaftliche Verantwortung und entdecken, dass ihr Engagement gerade für die Entwicklung von Städten und Regionen wichtig ist. Dieses Engagement bedeutet eine aktive Gestaltungsrolle von Unternehmen für attraktive und lebenswerte Standorte. Eine 2018 erschienene Studie der Bertelsmann Stiftung und des deutschen Stifterverbandes zeigt, dass die deutsche Wirtschaft ca. 9,5 Milliarden Euro im Jahr für das Gemeinwohl ausgibt, ein Betrag, der sogar die Gesamtsumme der privaten Spenden in Deutschland übersteigt (LABIGNE et al. 2018). Folglich scheint es für viele Unternehmen heute immer wichtiger zu sein, über ihre Grenzen hinaus zu agieren und eine aktive Rolle rund um den Unternehmensstandort einzunehmen. Damit soll die Attraktivität von Stadt oder Region erhalten oder im besten Falle gesteigert werden. Dieses (lokale) Unternehmensengagement für den Standort ist weder neu noch reiner Selbstzweck und hat bei vielen Unternehmen eine lange Tradition. Das trifft besonders auf Mittelständler und Familienunternehmen zu (SuwaLA 2019, Albers \& Suwala 2020a). Allerdings gibt es hinsichtlich der Ziele, Motive und Umsetzungen gerade in den letzten Jahren eine neue Dynamik. So wurde der Bereich gesellschaftliche Unternehmensverantwortung (Corporate Social Responsibility - CSR) inzwischen in vielen Unternehmen professionalisiert (z.B. öffentlich-private Partnerschaften statt klassischer Stiftungs-, Spenden- oder Sponsoring-Modelle) und auf den gesellschaftlichen Wertewandel und Nachhaltigkeitsdiskurse abgestimmt. Außerdem erfolgte in vielen Bereichen die Erweiterung dieser Verantwortung auf die räumliche Dimension bzw. wird diese anhand von einschlägigen regionalen oder lokalen Vorhaben nachvollziehbar. In der Wissenschaft wird dieses raumbezogene Handeln übergeordnet als Corporate Spatial Responsibility bezeichnet (CSpR) (Albers 2011, KNIELING et al. 2012, Harrison 2014, Albers \& Hartenstein 2017a, Albers \& Suwala 2018, Albers \& SUWALA 2020b).

Diese raumbezogene Unternehmensverantwortung ist aber auch vor dem Hintergrund der vielfältigen Herausforderungen in der Stadt- und Regionalentwicklung - insbesondere bezogen auf die Planung und Steuerung - zu betrachten. Gerade kleinere Städte und ländliche Regionen sind häufig nicht nur vom Strukturwandel betroffen, sie kämpfen 
mit finanziellen Engpässen, leiden unter Humankapital- und Kaufkraftabfluss, maroder oder schlecht ausgebauter Infrastruktur und abnehmender Attraktivität besonders in den Ortszentren. Für Unternehmen wird es an diesen kleineren und oft peripheren Orten deshalb immer schwieriger Fachkräfte zu finden oder zu binden. CSpR - Konzepte können hier Ansatzpunkte bieten und zu einer Förderung von lebenswerten, wettbewerbsfähigen und innovativen Standorten beitragen. Je nach Intensität des Engagements kann es sogar zur Etablierung sogenannter, alternativer, ortsspezifischer Organisationsund Führungsstrukturen seitens der Privatwirtschaft (englisch Place Leadership, PL) kommen. Diese Entwicklung lässt sich in einen generellen Trend einordnen: In Zeiten fortwährender Liberalisierung, Deregulierung und Privatisierung, begrenzter institutioneller Kapazitäten, fehlender Verwaltungseinrichtungen oder geringer Wirksamkeit von Behörden besteht ein größeres Interesse an nicht-staatlichem „ortsbezogenem“ bzw. einem ,place-based“ Engagement der Wirtschaft (HARRISON 2014, SuwALA et al. 2018). Durch die Kombination der beiden aufkommenden Diskurse über Place Leadership (PL) (Gibney et al. 2009, Collinge \& Gibney 2010, Beer \& Clower 2014, Liddle et al. 2017, Sotarauta et al. 2017) und Corporate Spatial Responsibility (CSpR) (Albers 2011, KNieling et al. 2012, Harrison 2014, Albers \& Hartenstein 2017a, Albers \& SuwaLa 2018) stellt dieser Beitrag Ansätze sowohl für ein unternehmensorientiertes und regionales Engagement (CSpR) als auch für privatwirtschaftlich getragene ortsspezifische Organisations- und Führungsstrukturen (PL) vor.

Dieser Beitrag soll primär die grundlegenden Inhalte der beiden Konzepte auf Basis der vorhandenen Fachliteratur vorstellen, eine kombinierte Sicht der beiden Themenstränge mithilfe von Fallbeispielen eröffnen und eine erste empirische Einordnung anbieten. Generelles Ziel dieser wissenschaftlichen Auseinandersetzung ist - neben einer Systematisierung dieses unternehmensorientierten regionalen Engagements - die Offenlegung von Strukturen der Ortsführerschaft innerhalb von CSpR Projekten, damit in der weitergehenden Forschung alternative Organisations- und Akteurskonstellationen für den praktischen Einsatz und zur Wirkungsoptimierung formuliert werden können. Erste Handlungsempfehlungen am Ende des Beitrags geben bereits einen kurzgefassten Ausblick auf diese Intention. Der nachfolgende Abschnitt fasst die aktuelle Forschungsdiskussion zu CSpR und Place Leadership zusammen. Dem schließt sich eine Zusammenführung der beiden Begriffe an. Im Abschnitt „Unternehmens- und Engagementlandschaften in Kleinstädten" - der eigentlichen Analyse - werden Fallbespiele auf Basis einer aktuellen Desktop Recherche und anhand von bisherigen Studien und Projektbeteiligungen der Autoren vorgestellt. Schließlich folgt neben der Einordnung und Systematisierung der Fallbeispiele innerhalb eines CSpR Modells, das Intensitäten dieses unternehmensorientierten regionalen Engagements aufzeigt und es potentiellen alternativen ortsspezifischen Organisations- und Führungsstrukturen zuordnet, eine Diskussion von Wirkungs- und Handlungsfeldern dieser Vorhaben und ein diskursorientierter Ausblick. 


\subsection{Corporate Spatial Responsibility}

Der Begriff Corporate Spatial Responsibility (CSpR) beschreibt raumbezogenes unternehmerisches Engagement, das über die wirtschaftlichen Kernkompetenzen von Unternehmen hinausgeht. CSpR kann somit als Erweiterung des allseits bekannten Konzepts der Corporate Social Responsibility (CSR) verstanden werden, welches vor allem auf das soziale und ökologische Engagement fokussiert ist (ALBERs 2011, ALBERS \& Suwala 2018). CSpR folgt somit den CSR-Grundsätzen, einschließlich Unternehmensethik und Corporate Citizenship, um gesellschaftliche Probleme anzugehen, die primär nicht mit den wirtschaftlichen Unternehmensaktivitäten verbunden sind (obwohl diese Maßnahmen für Unternehmensziele genutzt werden können) (Hiss 2006, Beschorner 2010, Hanson et al. 2010). CSpR wird in diesem Beitrag als Sammelbegriff für raumbezogenes Unternehmensengagement auf unterschiedlichen Maßstabsebenen (lokal, regional, städtisch, ländlich) (KNIELING et al. 2012) verstanden und schließt damit verwandte Konzepte wie „Corporate Regional Responsibility“ (CRR) (KIESE \& SCHIEK 2016, SCHIEK 2017), „Corporate Urban Responsibility“ (CUR) (Albers 2011, Albers \& HARTENSTEIN 2017a) oder „Corporate Regional Engagement“ (CRE) (Lengauer \& TÖDTLING 2010, BÜrCher \& MAYER 2018) mit unterschiedlichen räumlichen Zielsetzungen (z.B. Revitalisierung des baulichen Umfelds (AlBERs 2011), Förderung (nachhaltiger) und technologiebasierter Stadtentwicklung (SCHIEK 2017) mit ein.

Die Ziele, Instrumente und Organisationsformen für raumorientiertes Unternehmensengagement haben sich im Laufe der Zeit und im Zuge des wirtschaftlichen und gesellschaftlichen Fortschritts weiterentwickelt und ausdifferenziert. So geht es längst nicht mehr allein um die Bewältigung der ,sozialen Frage' durch das Engagement großer Industrieunternehmen (z.B. Krupps Margarenthenhöhe in Essen) im Rahmen des Werkswohnungsbaus oder Unternehmensschenkungen und Stiftungen zur Etablierung bürgerlicher Einrichtungen (ALBERS 2011). Vielmehr hat sich das Themenfeld in den letzten 20 Jahren - auch aufgrund gesamtgesellschaftlicher, ethischer und moralischer Ansprüche (Wertebewusstsein), stark entwickelt und professionalisiert. Obwohl herkömmliche CSR-Instrumente wie Spenden, Sponsoring und Stiftungen - um eine Raumwirkung erweitert - nach wie vor weit verbreitet sind (Albers \& Suwala 2018), werden zunehmend komplexe Organisationsformen wie öffentlich-private Partnerschaften, Kooperationen mit zivilgesellschaftlichen Akteuren und langfristige Projekte in Regionen und Städten mithilfe von Unternehmen umgesetzt (vgl. LABIGNE et. al. 2018, BRANDT et al. 2019). Mit diesen Entwicklungen ging auch eine Diversifizierung der Maßnahmen einher. Neben einfachen unternehmensgetriebenen sozialen, kulturellen oder erlebnisorientierten Bauprojekten (z.B. Kindertagesstätten, Museen) (Albers 2011), kommt es aber auch zu einer unternehmensgetriebenen Revitalisierung von Städten und Regionen - z.B. durch die Bereitstellung von privaten Flächen für die öffentliche Nutzung (KAYDEN 2000), die Verlagerung bzw. Reurbanisierung von 
Unternehmen (MozINGo 2011) oder der alternativen Umnutzung von Altindustriestandorten (z.B. Stadtteilerneuerung durch Kultur- und Kreativwirtschaft) (SuwaLA 2015, Kitzmann \& Suwala 2018). Darüber hinaus existieren aber auch weitreichende privatwirtschaftliche Ansätze wie bspw. Business Improvement Distrikte (BID) (HoYT 2003) oder raumbasierte Vermarktungsstrategien (z.B. Town Center Management) (Coca-StefaniaK et al., 2009). Schließlich sind sogar ganzheitliche, systemische Ansätze von CSpR mit hauptsächlich privatwirtschaftlich getriebenen Initiativen der städtischen oder regionalen Entwicklung (business-led communities, Einright et al. 2016) in Kleinstädten verbreitet (Albers \& Suwala 2018, Albers \& Suwala 2020b).

\subsection{Place based Leadership}

Das Konzept des „Place Leadership“, oder auf Deutsch übersetzt der „Ortsführung“ basiert auf einer Renaissance der räumlichen Dimension innerhalb der Leadership-Forschung (z.B. Judd \& Parkinson 1990, Stough 2003, Sotarauta 2005), wobei die Thematik erst seit dem Jahr 2010 wirklich an Bedeutung gewinnt (STIMsON et al. 2009, Collinge \& Gibney 2010, Sydow et al. 2011, Beer \& Clower 2014, Liddle et al. 2017, Sotarauta et al. 2017). Die Popularität beruht sowohl auf der fortschreitenden Liberalisierung, Deregulierung und Privatisierung vormals staatlicher Aufgaben und Wirtschaftssektoren als auch auf dem Boom der Wissen- und Informationsökonomie (GIBNEY et al. 2009). Beide Umstände führten zu einem beschleunigten wirtschaftlichen, technischen und wissenschaftlichen Fortschritt sowie einer stärkeren Abhängigkeit von Akteuren, Institutionen und Branchen, die insbesondere auf Wissensproduktion ausgelegt sind. Eine Folge war die Etablierung von neuen überinstitutionellen Zusammenschlüssen (z.B. Public-Private-Partnerships, BrinkerHOFF \& BRINKERHOFF 2011) oder regionalen Bündnissen zwischen bis dahin kaum gemeinsam agierenden Interessensgruppen (Wirtschaft, Wissenschaft, Politik) (z.B. Technologieparks, SuwaLA \& DANNENBERg 2009). Das gemeinsame Ziel war und ist die Förderung von Kreativität, Innovation und Unternehmertum (z.B. Etzkowitz \& LeYdesdorfF 2000, Suwala 2014, BRINKHOFF et al. 2015) durch neuartige Führungskonstellationen in verschiedenen räumlichen Kontexten (z.B Stadt / Land, z.B. globaler Norden und globaler Süden: BEER \& Clower 2014, ANderton 2017, Hu \& Hassink 2017). Vor diesem Hintergrund kann der Begriff des ,Place Leadership“ bzw. der Ortsführung wie folgt beschrieben werden: „the tendency of the community to collaborate across sectors (...) encompassing the public, private and civil““ (STIMSON et al. 2002, p. 279) einschließlich „of overlapping realms (...) in a given locality“ (HAMBLETon 2015, p. 137). Den Zusammenschlüssen und Führungskonstellationen sind dabei grundsätzlich keine Grenzen gesetzt (NICOLDS et al. 2017). Die ehemalige zumeist staatliche oder hoheitliche Aufgabe der Orts- oder regionalen Führung kann so auch durch andere Stakeholder wie Hochschulen (RAAGMAN \& KeERBER 2017), gemeinnützige Organisationen (PotLUKA et al. 2017) oder öffent- 
liche Unternehmen (Rossiter \& SMITH 2017) mit unterschiedlichen Zielen wie z.B. nachhaltiger Entwicklung (HorLINGS \& PADT 2013) übernommen werden. Trotz der bisher gesammelten Erkenntnisse ist bei weitem nicht klar, was ,effektive Ortsführung“ / „effective Place Leadership“ in der konkreten Ausgestaltung bedeutet. Vor allem bleiben Fragen offen, wer führen soll (z.B. öffentlich, privat oder zivil), HAMBLETon 2011), warum „Place Leadership“ notwendig ist (z.B. Rückzug des Staates, SotaraUta et al. 2017, Suwala et al. 2018), zu welchem Zweck Place Leadership benötigt wird (z.B. Zukunftsvisionen, Stimson et al. 2009), wann (z.B. Transformation von Regionen, Suwala \& MiceK 2018), wo eine solche Führung erforderlich ist (z.B. Führung in / von (strukturschwachen) Regionen, Collinge \& Gibney 2010, Albers \& Suwala 2018), und wie man führt (z.B. formales vs. informelles Place Leadership, SotARAUTA et al. 2012).

Da sich dieser Beitrag auf die privatwirtschaftliche oder unternehmensorientierte Führung und deren Beitrag zur Stadt-/Regionalentwicklung konzentriert wird in Folge auf diesen Teilbereich der Ortsführung abgestellt. Privatwirtschaftliche oder unternehmensorientierte Ortsführung ist nicht neu, wie zahlreiche Beispiele zeigen. Die Idee hat eine lange Tradition in sogenannten Firmenstädten/ Company Towns (z.B. Siemensstadt im Nordwesten Berlins (Deutschland), American Railway Union, Pullman, IL (USA), Volkswagen in Wolfsburg (Deutschland), Lego in Billund (Dänemark)) (IDRIS-Soven et al. 1978, GARNER 1992). Obwohl das Hauptziel dieser Art von „Place Leadership“ darin besteht, „die wirtschaftliche Leistung oder das wirtschaftliche Umfeld der Region zu verbessern" (STIMson et al. 2002, 279) (neben kulturellen, sozialen und ökologischen Aspekten), unterscheidet sie sich nicht wesentlich von anderen oder gemischten Konstellationen (z.B. im Zusammenarbeit mit der Zivilgesellschaft) des Place Leadership. Die teilweise schlechten Erfahrungen, die im Rahmen von Company Towns (z.B. Abhängigkeit, Ausbeutung, Machtkonzentration etc.) gemacht wurden und die de facto von Unternehmen monopolisierten Führungsstrukturen führten dazu, dass das Interesse an unternehmensgetriebener „Ortsführung“ insbesondere in der zweiten Hälfte des 20.Jahrhunderts nachließ (z.B. siehe für die USA, HANson et al. 2010). Darüber hinaus fußt das gegenwärtige Verständnis von „Place Leadership“ eher auf gemeinschaftlich eingebrachten, heterarchischen und sektorenübergreifenden Ressourcen (öffentlich, privat, zivil) als auf der Grundlage ,einer großen Person oder eines großen Unternehmens" mit einer dominanten Position wie es häufig früher der Fall war (STIMson et al. 2002, BEER \& CLOWER 2014). Es ist jedoch keineswegs klar, ob eine eher oligarchische Ortsführung (Belland et al. 2018) oder eine gemeinschaftliche Führung von Unternehmen in Regionen (STIMson et al. 2002) effektiver ist. Letzteres hängt von vielfältigen Faktoren wie dem Führungsstil, Macht, Vertrauen und/oder Zusammenarbeit der beteiligten Akteure ab (STIMSON et al. 2009, HAMBLETON 2011). 


\subsection{Zusammenführung von CSpR und Place Leadership}

Vor diesem Hintergrund ist das Ziel des Beitrags, Erkenntnisse aus dem Place Leadership und dem CSpR Konzept für einen neuen Ansatz der privatwirtschaftlich-orientierten Ortsführung in Wert zu setzen. Dabei sind die hier gemachten Formulierungen Teil einer umfassenden Forschungsarbeit zum regionalen, unternehmensorientierten Engagement und bilden deshalb nur einen ersten Ausschnitt ab.

Um Beziehungen, Überschneidungen oder auch Gegensätze der beiden Konzepte zu identifizieren und den Mehrwert einer Zusammenführung für die fachwissenschaftliche Diskussion herauszuarbeiten, wurde die einschlägige Literatur zu Place Leadership und CSR/ CSpR gesichtet und analysiert. Folgende Leitfragen standen dabei im Vordergrund: Kann eine privatwirtschaftliche / unternehmensorientierte Ortsführung CSpR Maßnahmen optimieren? Oder umgekehrt: Können CSpR Maßnahmen die Ausgestaltung einer privatwirtschaftliche Ortsführung verbessern bzw. etablieren? Welche CSpR Maßnahmen bedingen Place Leadership, um eine Wirkung zu erzielen? Gibt es unterschiedliche Intensitäten von Place Leadership und CSpR und wie verhalten sich diese zueinander? Bisher können aus der laufenden Forschung dazu folgende Aussagen getroffen werden: Ortsbezogenes, unternehmerisch getriebenes oder privat-öffentliches Place Leadership ist ein altes Phänomen, welches mit unterschiedlichen Einsatzmöglichkeiten, Praktiken, Instrumenten und Intensitäten des Engagements - je nach institutionellem Rahmen und Wirtschaftssystem - einhergeht (STIMsON et al. 2009, HANson et al. 2010, Sotarauta 2016). Obwohl die Übertragung von Konzepten auf neue Anwendungsbereiche nicht immer unproblematisch ist, kann von einer gegenseitigen Befruchtung zwischen den Diskursen um Place Leadership und CSpR ausgegangen werden (Albers \& Suwala 2020b).

Aber nähern wir uns zunächst dem Verhältnis zwischen herkömmlicher CSR und Führung. Obwohl die beiden Konzepte miteinander verbunden werden könnten, ist bislang nur wenig über den Zusammenhang zwischen Leadership und der Umsetzung von CSR bekannt (DE RUITER et al. 2018, 871); außerdem haben empirische Studien zu CSR die Funktion der Unternehmensleitung bei der Umsetzung von CSR weitgehend ignoriert (Waldman \& Siegel 2008). Obwohl es Studien gibt, die sich mit dem Zusammenhang zwischen Leadership und CSR im Allgemeinen im Sinne eines ,socially responsible leadership“ befassen (z.B. VAN VELSOR 2009), ist bisher wenig über die Raumkomponente in dieser Relation bekannt. In Zeiten wachsender interaktiver und gemeinschaftlicher Governance-Modelle muss aber auch der Frage nach einer sozial verantwortenden Führung und ihrem Kontext, insbesondere auf regionaler oder kommunaler Ebene, mehr Aufmerksamkeit geschenkt werden. In diesem Feld trifft Führung / Leadership auf Regionen, Städte oder ländliche Kommunen, und sie wird gleichzeitig von diesen räumlichen Einheiten beeinflusst (BEer \& Clower 2014, Sotarauta et al. 2017). DevinNey fordert sogar ein Umdenken: „rethinking the meaning of space and place (...) as the rise of CSR is part and parcel of a more general phenomenon that 
is redefining the fundamental meaning of sociopolitical and economic geography. As corporations and economies have globalized, the sociopolitical structures have lagged behind" (DevinNey 2011, pp. 329, 339). Bisher gibt es nur sehr wenige Studien, die den Zusammenhang zwischen Place Leadership und CSpR (un-)mittelbar aufgreifen (Voegtlin et al. 2012, KNieling et al. 2012, Albers \& Suwala 2020b). In einer dieser Arbeiten wird beispielsweise das privatwirtschaftliche Engagement als ,proaktive/r Unternehmer/in mit Ortsführerschaft" bezeichnet. Dabei geht es um ganzheitliche und systemische CSpR-Maßnahmen, z.B. die Umsetzung von durch Unternehmen initiierten Masterplänen oder privat-öffentlich getragene Maßnahmen zur (stadt-)räumlichen Entwicklung (Albers \& Suwala 2018).

Auf diesen komprimierten Zwischenstand aufbauend wird nachfolgend der Fokus auf das Unternehmensengagement in Kleinstädten und dem ländlichen Raum gerichtet und anhand von einigen Beispielen der CSpR / Place Leadership Diskurs vertieft. Dabei muss angemerkt werden, dass diese räumliche Fokussierung innerhalb des CSpR und PL Diskurses erst am Anfang steht, allerdings übergeordnete Anknüpfungspunkte bestehen: Etwa in der Suche nach Lösungsansätzen und Kooperationsmodellen zur Entwicklung von Kleinstädten in peripheren Lagen (siehe Publikation zum BBSR ExWoSt Projekt „Potenziale von Kleinstädten in peripheren Lagen“ 2016, BBSR 2016) oder der Rolle von Hidden Champion Unternehmen in der Stadtentwicklung (BBSR Forschungsprojekt „Hidden Champions - Stabilisierungs- und Entwicklungsfaktoren von Kleinstädten in peripheren Lagen“ 2017-2019, LANG et al. 2019) um nur zwei aktuelle Beispiele zu nennen. Einen weiteren Anschub für diesen thematischen Schwerpunkt liefert eine Anschlussstudie des CC-Survey des deutschen Stifterverbandes und der Bertelsmann Stiftung, welche den Fokus auf das regionale Engagement von Unternehmen richtet (LABIGNE et al. 2019). Die darin erkannte Kernaussage, dass Unternehmensengagement sich zu einem Großteil auf die Förderung der Region bezieht, fördert die wissenschaftliche Auseinandersetzung gerade hinsichtlich der Synergien aus Unternehmensengagement und Regionalentwicklung (vgl. BäUMER et al.2019).

Im Rahmen unseres Beitrags möchten wir nun einen Zusammenhang zwischen CSpR und Ortsführung (Place Leadership) herstellen. Dabei schlagen wir ein sogenanntes Reifegradmodell vor, welches in Form einer Pyramide aufgebaut ist. Hierbei können nicht nur unterschiedliche Intensitäten von CSpR-Maßnahmen abgebildet, sondern diese Maßnahmen auch möglichen Implikationen im Zusammenhang mit Ortsführung zu geordnet werden (vgl. Abb.1, Seite 77). Das Modell wurde ursprünglich von SCHNEIDER (2012, p. 29) für die Tragweiten herkömmlicher CSR-Maßnahmen entwickelt und von Albers und Suwala (2018) auf räumliches Unternehmensengagement übertragen. Dabei wird nach vier Intensitäten von CSpR differenziert, die sich je nach „Grad der Verantwortung / Raumbezug" unterscheiden: CSpR 0.0 und CSpR 1.0 umfassen Aktivitäten des Unternehmensengagements, welche einem konventionellen Engagement gleichen, sich ohnehin aus der Einhaltung von rechtlichen Rahmenbedingungen und / oder der Verfolgung von Unternehmenszielen ergeben, eher zufällige Auswirkungen haben (CSpR 0.0) oder einem philanthropischen Engagement zuzuschreiben sind 
(CSpR 1.0). Der Raumbezug ist vielfach Beiwerk und die räumlichen Auswirkungen eher passiv bzw. ein Zufallsprodukt. Während CSpR 2.0 bereits einen intendierten und systemischen Rahmen besitzt, welcher gezielt auf regionale, wirtschaftliche und gesellschaftliche Synergien zwischen der Stadt / Region und dem Unternehmen abzielt, greift CSpR 3.0 gar aus unterschiedlichen Gründen proaktiv in (ehemals) hoheitliche Aktivitäten des Staates (z.B. Ämter oder Behörden) ein. Sowohl CSpR 2.0 als auch CSpR 3.0 Maßnahmen können Elemente einer ortsbezogenen Führung (z.B. Entwicklung von Masterplänen) - also „Place Leadership“ - beinhalten und stehen damit an der Spitze der Reifegrad-Pyramide (Albers / SuwALA 2020b).

\subsection{Unternehmerische Engagement- und Führungsland- schaften in Kleinstädten}

In Kleinstädten finden sich häufig ortsprägende mittelständische Unternehmen - oft Familienunternehmen, mitunter sogar Unternehmen, die als Hidden Champions charakterisiert werden können (VonNAHME et al. 2018, LANG et al. 2019, BASCO \& Suwala 2020). Die Merkmale dieser Unternehmen sind nicht nur, dass sie in der Regel auf bestimmten Nischenmärkten europa- oder weltweit führend, nicht börsennotiert, familiengeführt und als solche der Öffentlichkeit weitgehend unbekannt sind (SIMON 1996), sondern dass sie sich durch eine langfristige Verwurzelung und Verbundenheit maßgeblich für die jeweilige Stadt oder Region engagieren (BASCO et al. 2020). Dieses raumbezogene, unternehmerische Engagement kann grob als Fähigkeit bezeichnet werden, regionalwirtschaftliche Rahmenbedingungen, Netzwerke und Governancestrukturen zu beeinflussen (LENGAUER \& TöDTLING 2010). Insbesondere in Kleinstädten geht es dabei um eine unternehmens- und sektorenübergreifende, langfristige und zum Teil institutionalisierte Zusammenarbeit, die sowohl einen Nutzen für die gesamte Stadt/Region stiften als auch die Wettbewerbsfähigkeit der Stadt/Region stärken soll. Darin sind konkrete, wenn auch sehr verschiedenartige Maßnahmen zur Verbesserung der Bildungslandschaft (z.B. Schulen) oder der Infrastruktur (z.B. Wohnungsbau, Verkehrsanbindungen) enthalten, bei denen Nachholbedarf besteht (BÜRCHER \& MEYER 2018, BRANDT et al. 2019). So sollen Fachkräfte etwa durch neue Werkswohnungen oder Investitionen in Freizeit- und Sozialeinrichtungen gewonnen und Nachbarschaften unterstützt werden, damit der Mitarbeiternachwuchs vor Ort gesichert ist und nicht in Großstädte abwandert. Viele dieser Unternehmen erkennen zudem, dass sie im globalen Wettbewerb nicht nur die Handlungsoption des Standortwechsels besitzen, sondern zunehmend auch die Möglichkeit der Standortgestaltung haben (Albers \& Suwala 2018). So können Innenstädte belebt oder Maßnahmen zur Steigerung der Lebensqualität gefördert, letztlich Standortfaktoren beeinflusst werden. Gleichzeitig zeichnet sich dieses Unternehmensengagement in kleinen Städten durch Besonderheiten aus und bildet durch eine überschaubare Anzahl an handelnden Akteuren ortsspezifische 
Organisations- und Führungsstrukturen. Folglich kommt es naturgemäß zu einer Fokussierung auf Einzelpersonen oder Schlüsselfiguren, die als neue ,Dirigenten' gleich in mehrfacher Rolle als Leader, Intermediär oder Governance-Pionier auftreten können (GAILING \& IBERT 2016). In ortsbezogenen Führungsprozessen machen diese Schlüsselpersonen zwar einen entscheidenden Unterschied aus, sollten aber weder heroisiert noch isoliert, sondern in einem kleinstädtischen Orchester unterschiedlicher Akteursgruppen betrachtet werden (ALBERS \& SuwALA 2018). Dieses kleinstädtische Unternehmensengagement unterscheidet sich deswegen auch oft im Vergleich zur Großstadt und ist als solches nicht ohne Weiteres übertragbar. Denn gerade (zivil-) gesellschaftliche-, politische und zuletzt auch wirtschaftliche Strukturen sind auf Kleinstadtebene meist stärker durch informelle Kontakte, „kurze Wege“, schlanke Strukturen und persönliche Beziehungen gekennzeichnet. Gleichzeitig bremsen fehlendes Know-How, eine geringe personelle oder finanzielle Ausstattung, eingeschränkte politische Einflussnahme, planerische Steuerung oder die Dominanz weniger Personen die Entwicklungspotentiale von Kleinstädten. Sicht- und erfahrbar werden diese teils ambivalenten Entwicklungen an konkreten Projekten, die im Folgenden vorgestellt werden. Dabei werden einerseits die Relevanz und der Diskurs aufgezeigt, andererseits Inhalte für die anschließende Einordnung in das Reifegradmodell formuliert.

\section{Engagement für soziale- und kulturelle Infrastruktur}

Eine Form des herkömmlichen Unternehmensengagements im Kontext der Stadtund Regionalentwicklung ist die Finanzierung oder Förderung von Gebäuden für gesellschaftliche, bildende oder soziale Zwecke. Im 19. Jahrhundert verhalf das Spenden für Kunst- und Kulturbauten den Städten zu einer neuen, bürgerlich geprägten Identität. Diese Tradition hat sich bis heute erhalten und birgt neben Prestige für die Mäzene auch einen Aufwertungseffekt für Städte und Regionen. Die Wirkung wird seit den 1990er Jahren als »Bilbao-Effect« betitelt. Der Begriff beschreibt die Strategie, mit einem symbolhaften Bauwerk eine Stadt wirtschaftlich und kulturell zu beleben, aus ihrer Abseitsposition und ökonomischen Krise zu befreien beziehungsweise auf der globalen »touristmap « zu positionieren (PlazA 2008). Im deutschen Mittelzentrum Herford beispielweise wurde durch eine Unternehmensinitiative das Marta Kunstmuseum (mit Hilfe des Architekten Frank Gehry, der durch das Guggenheimmuseum in Bilbao auch maßgleich zum gleichnamigen Effekt beisteuerte) gebaut (Eröffnung 2005). Andernorts werden die gesamten Kosten für die Kultur- bzw. Kunstbauten komplett von Einzelunternehmen getragen. Besonders in kleineren Städten prägen Kulturbauten einzelner Familienunternehmen oder Hidden Champions die Zentren: beispielhaft ist das Würth Kunstmuseum in Künzelsau, die Galerie Stihl in Waiblingen, die Kunsthalle Weishaupt in Ulm, das Museum Barberini in Potsdam (Hasso Plattner, Mitgründer SAP) oder das Knauff Museum in Iphofen. All diesen Projekten liegt eine starke Bindung der Unternehmenspersönlichkeiten an den Standort zugrunde (meist Familienunternehmen); das Motiv für das Engagement ist dabei aber eher mäzenatisch geprägt als einer gesamtgesellschaftlichen unternehmerischen Verantwortung zuzuordnen (ALBERs 2011). Die 
Stärkung von Stadtimage und Belebung der Ortszentren hat allerdings in den letzten Jahren eine zusätzliche Rolle hinsichtlich der Erhaltung und Anwerbung von Mitarbeitern gewonnen und damit auch das Spektrum für Unternehmensengagement erweitert. Das betrifft etwa den Bau von Kindertagesstätten oder Bildungseinrichtungen, die mit unternehmerischer Unterstützung zügig umgesetzt werden können. Die Firma Stihl baut seit 2019 eine Kindertagesstätte für die Kinder der Mitarbeiter und Bürger der Stadt Waiblingen in Kooperation mit der Kommune. In Künzelsau finanziert die Firma Würth den Bau einer Hochschule, an der potentielle Mitarbeiter für das Unternehmen und für die Region ausgebildet werden.

\section{Engagement für Wohnraum - Werkswohnungsbau 2.0}

Der Wohnraummangel stellt in vielen (Groß-)städten eine wachsende Problematik dar. Das erkennen auch immer mehr Unternehmen, deren Mitarbeiter Probleme haben, eine Wohnung nahe der Arbeitsstätte zu finden. Betroffen sind vor allem Angestellte in unteren, aber auch mittleren Einkommensgruppen, besonders jedoch Auszubildende. Zudem sorgen sehr gut bezahlte Fachkräfte etwa aus der High-Tech-Wirtschaft für Verdrängungseffekte auf lokalen Wohnungsmärkten (z.B. SAP-Mitarbeiter in Walldorf/Baden-Württemberg). Deshalb erfährt der Werkswohnungsbau gerade eine Renaissance. Diese Renaissance ist aber inzwischen auch in kleinen Städten und dem ländlichen Raum sichtbar. So sind es längst nicht mehr nur Tech-Konzerne wie Google oder Facebook neben Unternehmen wie BASF, den Münchner Stadtwerken oder VW, die wieder in den Wohnungsbau einsteigen. Die Deutsche Bahn hat angekündigt, im bayerischen Baldham ein Mitarbeiterwohnhaus mit integrierter Gemeindebücherei zu errichten. In Memmingen werden von der Bäckerei Häussler am Firmensitz Mitarbeiterwohnungen geplant. Diese Beispiele sind dem prosperierenden Ballungsraum München und der ausstrahlenden Wohnungsproblematik zuzurechnen. An peripheren, ländlichen Standorten sind Unternehmen jedoch mittlerweile auch im Wohnungssektor aktiv, etwa weil der lokale Wohnungsmarkt nachgefragte Wohnungstypen nicht bietet. Gerade im Tourismus oder in der Landwirtschaft werden Wohnungen für Saisonkräfte benötigt; andernorts geht es darum, Wohnungen mit städtischen Qualitäten für Fachkräfte bereitzustellen. Das dient auch der Vermeidung von langen Pendlerwegen und schließlich als Maßnahme zur Vereinbarkeit von Beruf und Familie.

\section{Engagement für Stadtzentren / -quartiere - Unternehmen als Impulsgeber}

Viele Städte und Gemeinden kämpfen mit der Verödung ihrer Zentren - Einzelhandel in traditionellen Geschäftsstraßen befindet sich auf dem Rückzug oder wird an nicht-integrierten Standorten (z.B. Shopping Centern) konzentriert und das Gewerbe siedelt sich in Gewerbegebieten am Rand an. Anders im bayerischen Mertingen (knapp 4000 EW. Landkreis Donau-Ries). Die Ortsmitte soll in den nächsten Jahren ein neues Gesicht bekommen. Dabei spielt die Firma Zott (ein 1926 gegründetes Familienunternehmen mit ca. 3.000 Mitarbeitern und eine der führenden Molkereien Europas) eine 
wichtige Rolle. Unterstützt mit dem Modellvorhaben „Ort schafft Mitte“ seitens des Bauministeriums des Freistaats Bayern soll das Zentrum wieder zu einem lebendigen und attraktiven Mittelpunkt der Gemeinde entwickelt werden. Die Firma Zott errichtet hier ihre neue Verwaltung und trägt damit zur Belebung der Innenstadt bei. Außerdem wurden mit dem Programm weitere Entwicklungen im Ortszentrum auf den Weg gebracht, wie etwa der Umbau des zentralen Dorfplatzes oder die Sanierung angrenzender Gebäude. Was der Trend zu Unternehmenscampussen in großen Städten zeigt (AlBers \& HARTENSTEIN 2017b), wird hier im Kleinen umgesetzt und als zentrumsstärkende Maßnahme erkannt. Die Firmenzentrale mit Cafeteria und Veranstaltungsräumen ist in das Dorfplatzkonzept eingebunden und sorgt neben den benachbarten öffentlichen und privatwirtschaftlichen Angeboten für eine neue Belebung des Ortskerns.

\section{Engagement für strategische Stadt- / Regionalentwicklungsprozesse-Masterplanin- itiativen}

Auf Initiative und mit Finanzierung der lokalen Wirtschaft wurden in Köln, Offenbach und Mönchengladbach während der letzten Jahre Masterpläne entwickelt. Erklärtes Ziel: Die Steigerung der städtischen Attraktivität und der ökonomischen Standortbedingungen. Privatwirtschaftlich initiierte Masterplaninitiativen gibt es aber nicht nur in Großstädten, sondern beispielsweise im niedersächsischen Duderstadt (ca. 22.000 Einwohner). Das Projekt „Duderstadt2020“ war eine Initiative des Unternehmers Hans Georg Näder, Firmeninhaber der Otto Bock GmbH, Weltmarktführer für Orthopädietechnik. Das Vorhaben wurde seit 2009 von einem Team der Hochschule für Angewandte Wissenschaft und Kunst Hildesheim, Holzminden und Göttingen (HAWK) entwickelt und organisiert. Ziel war, Maßnahmen zur qualitativen Stadtentwicklung in Form eines Masterplans anzuregen. Dabei wurden vor allem Konzepte entwickelt, die der Abwanderung von Fachkräften und dem demografischen Wandel entgegenwirken sollten. Diskussionsforen und Zukunftswerkstätten als integrative Instrumente trugen dazu bei, dass viele Bürger, Unternehmen und schließlich die Stadtverwaltung diesen Prozess mitgetragen haben. Die Duderstadt2020 GmbH \& Co. KG wird heute als wirtschaftlich und rechtlich unabhängige Stadtentwicklungsagentur mit sechs Themenfeldern betrieben. Zuletzt wurden die Stadtvision Duderstadt 2030 und der Masterplan „Futuring Duderstadt“" entwickelt (<www.duderstadt 2030.de>).

\section{Flankierende Maßnahmen durch Dritte - Netzwerke \& Bündnisse schaffen, Engagement stärken}

Stadtentwicklung wird immer mehr als Gemeinschaftsaufgabe verstanden, die von Wirtschaft, Wissenschaft, Zivilgesellschaft/Bürgern und Politik/Verwaltung - als Quadruple Helix - getragen wird (LANGe et al. 2011). CSpR bedeutet deshalb zunehmend auch die Förderung von Engagement oder sozialen Strukturen im Rahmen eines vernetzten und strategischen Handelns. Beispielhaft für diesen Trend ist die Initiative „Engagierte Stadt“, ein kooperatives Projekt von Bertelsmann Stiftung, BMW 
Stiftung Herbert Quandt, Herbert-Quandt-Stiftung, Körber-Stiftung, Robert Bosch Stiftung, Generali Zukunftsfonds und dem Bundesministerium für Familie, Senioren, Frauen und Jugend. Die „Engagierte Stadt“ setzt auf lokale Zentren für Engagement und deren Potenzial, systematisch Engagement vor Ort zu entwickeln und zu fördern. 2015 wurden aus 272 Bewerbungen 50 Engagierte Städte für das Netzwerkprogramm ausgewählt und mit Fördergeldern und Beratungsleistungen unterstützt (www.engagierte stadt.de). Bereits seit 2007 ist der von der Bertelsmann Stiftung gegründete Verein „Unternehmen für die Region“ aktiv und wurde mittlerweile in das Projekt „Regionale Unternehmensverantwortung“ / die Plattform „Regional Engagiert“ $(<\mathrm{https} / / /$ regionalengagiert.de $>$ ) integriert, die mittelständische Unternehmen bzgl. Ihres Engagements vernetzt und über Projekte informiert ( $<$ https://www.bertelsmann-stiftung.de/de/unsereprojekte/unternehmensverantwortung-regional-wirksam-machen/did/122/>). Die Robert Bosch Stiftung fördert mit ihren Programmen „Actors of Urban Change“ und „Neulandgewinner" eine nachhaltige und partizipative Stadtentwicklung und ermöglicht, Kompetenzen für transsektorale Zusammenarbeit zu stärken (www.bosch-stiftung.de/ de/projekt/actors-urban-change, www.neulandgewinner.de). Weitere Beispiele sind das Programm Lokalhelden, das - gefördert von der schweizer Drosos Stiftung - Gründerprojekte im ländlichen Raum unterstützt ( $<$ www.lokalhelden-werden.de $>$ ) oder das Netzwerk Immovielien, das von der Montag Stiftung Urbane Räume mitinitiiert und gefördert wird ( $<$ www.netzwerk-immovielien.de $>$ ).

Neben den ausgewählten Beispielen sind selbstverständlich noch weitere gemeinschaftliche Zusammenschlüsse denkbar, so wie beispielsweise auch das gemeinsame Wirken von Bürgern und Unternehmern in Bürgerstiftungen.

\subsection{Diskurs und Einordnung der Initiativen in das Reife- gradmodell}

Die hier ausgewählten Initiativen und /oder flankierenden Maßnahmen durch Dritte sind aber nur ein Teil der Vorhaben in Kleinstädten, in denen sich Unternehmen oder die Privatwirtschaft engagieren. Die Beispiele wurden so gewählt, um hier ein möglichst breites Spektrum von Intensitäten des räumlichen Unternehmensengagements aufzuzeigen und diese dann bestenfalls auf Qualitäten der Ortsführung zu prüfen.

Das Engagement für soziale- und kulturelle Infrastruktur umfasst ein Bündel unterschiedlicher Maßnahmen, die zumeist im herkömmlichen CSR-Bereich angesiedelt sind und in ihrer großen Zahl eher - wenn überhaupt einen indirekten Raumbezug aufweisen (z.B. philanthropisches Engagement für Kindergärten und soziale Einrichtungen, z.T. als vereinbarte Ausgleichmaßnahmen etc.) (CSpR 0.0). Die Umsetzung von Kindertagesstätten oder Bildungseinrichtungen in Kooperation mit den Kommunen gelingt nur mit entsprechend langfristigen Organisationsstrukturen (CSpR 1.0). Ähnlich gelagert ist 
das Engagement für Wohnraum. So wirken Werkswohnungsbauprojekte entspannend auf den lokalen Wohnungsmarkt und sind somit eine positive Stadtentwicklungskomponente, sie verfolgen allerdings primär reaktiv das Ziel, Wohnraum für die eigenen Mitarbeiter anzubieten (CSPR 1.0, sind diese z.B. zudem in Quartierentwicklungskonzepte eingebunden kann von CSpR 2.0 gesprochen werden). Eine besondere Rolle nehmen seit etwa 20 Jahren ikonische Bauten für Kunst und Kultur ein, die oft durch Mäzenatentum und persönliche Motive der stiftenden Unternehmer geprägt sind. Diese Kunst- und Kulturbauten können innerhalb eines städtischen oder regionalen Gesamtkonzepts als Leuchttürme für die Stadt wirken und Orte(-szentren) neu beleben (sog. Bilbao-Effekt) (CSpR 1.0). Neu ist allerdings, dass diese Maßnahmen verstärkt eine räumliche Interpretation erfahren und sich explizit an der Förderung von Stadt oder Region orientieren oder sogar eine Rolle in der (Innen-) stadtaktivierung übernehmen (je nach Umfang und Einbindung in Planwerke, CSpR 1.0 bis CSpR 3.0). Alle diese Arten des Engagements zeichnen sich vor allem durch fragmentierte unternehmensgetriebene Interventionen aus, die insbesondere Partikularinteressen vertreten und entweder mit klassischen philanthropischen Maßnahmen oder Synergien durch Unternehmensziele einhergehen. Der räumliche Bezug schwingt zum Teil mit und ergibt sich aus der Notwendigkeit der Zusammenarbeit z.B. mit Kommunen. Von einer Ortsführung im Sinne des Place Leadership kann hier in der Regel nicht gesprochen werden.

Im Gegensatz dazu hat sowohl das Engagement für Stadtzentren / -quartiere mit Unternehmen als Impulsgeber als auch das Engagement für strategische Stadt- / Regionalentwicklungsprozesse in Gestalt von Masterplaninitiativen einen systemischen und oftmals auch ganzheitlichen Charakter, der in der Regel gezielt auf regionalwirtschaftliche und gesellschaftliche Synergien abzielt oder gar (ehemals) hoheitliche Aufgaben des Staates übernimmt. Dazu bedarf es meist einer mittel- oder langfristigen Ausrichtung nebst überinstitutionellen Kooperationen und Organisationsstrukturen. Entsprechend sind die Beispiele aus diesem Bereich meist höher im Reifegradmodell anzusiedeln, ferner ergeben sich hier berechtigte Fragen nach Aspekten der Ortsführung. Das Vorhaben „Zurück in die Mitte“ in Mertingen ließe sich wohl am ehesten als ein CSpR 2.0-Vorhaben einordnen, bei dem eine bewusste Systematik vorliegt, die darauf abzielt, aus einer „Win-win-Situation“ sowohl ökonomische als auch gesellschaftliche Synergien in der Stadt zu mobilisieren - ohne sich die stadtplanerischen Planund Rahmenwerke anzueignen. Für solch ein Projekt sind öffentliche Programme als Anschubhilfe und Know-How als Ressourcen nahezu unablässig. Obwohl hier eine Ortsführung nicht explizit intendiert ist, könnte sich diese aus der gelebten Realität ergeben, wenn beispielsweise öffentliche Nutzungen in den Gebäuden der neuen Unternehmenszentrale angesiedelt werden.

Das Projekt „Duderstadt2020“ mit seiner Masterplaninitiative ist sicherlich ein Ansatz, welcher weitgehend in die (ehemals) hoheitlichen Gebiete des Staates/ der kommunalen Verwaltung mit Bezug auf Stadtplanung eingreift. Dieses CSpR 3.0-Vorhaben ist progressiv, wirft aber auch zahlreiche Fragen auf. Sollte sich ein Unternehmen soweit in die Stadt- und Regionalplanung einmischen? Kann hier wirklich noch von 
einer gemeinwohlorientierten Planung gesprochen werden? Lässt sich Altruismus vom Selbstzweck trennen, und ist das überhaupt notwendig? Dabei kann wirklich von einer unternehmens- bzw. teilweise unternehmerinduzierten Ortsführung gesprochen werden, wo unter anderem herausragende Einzelpersonen meist eben aus Familienunternehmen eine Führungsrolle aus Leidenschaft und Verbundenheit für den Ort übernehmen (vgl. auch Albers \& Suwala 2020a). Gleichzeitig ist eine solche Rolle gerade auf der Kleinstadtebene mit Dominanz- und Abhängigkeitsverhältnissen verbunden. Fällt das Engagement des Unternehmens wegen ökonomischer Schwäche, Führungsnachfolge oder Änderung der Eigentümerverhältnisse (z.B. Absentee Ownership) aus, leidet der Standort entsprechend.

\section{Abb. 1: CSpR Reifegradmodell und potentielle Implikationen für eine Orts- führung (in Anlehnung an ALBERS \& Suwala 2018, p. 55, vgl. SCHNEIDER 2012, p. 29)}

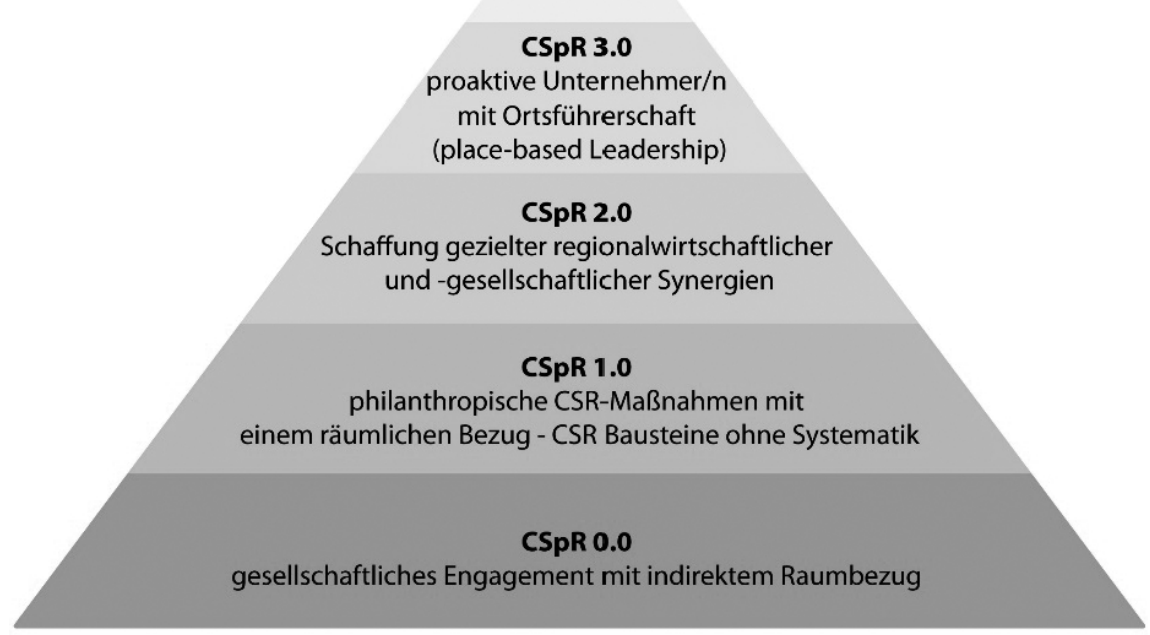

Schließlich sind die flankierenden Maßnahmen durch Dritte im Sinne der Engagementförderung, Vernetzung und Organisationsunterstützung (Bertelsmann, Robert Bosch Stiftung, u.a.) wie der Name schon sagt als flankierend oder begleitend zu betrachten. Diese Maßnahmen setzen den Fokus vor allem auf eine wirkungsoptimierende Beratungsleistung und Förderung von Unternehmensengagement und zivilgesellschaftlicher Akteure oder die Bildung von Netzwerken in Städten und ländlichen Regionen zwischen diesen Akteuren. Die engagierten Unternehmen oder Unterneh- 
mensstiftungen handeln in diesem Fall eher indirekt als Intermediäre oder Initiatoren, denn als Schlüsselakteure mit einer regionalen Einbettung wie sie etwa durch einen Firmensitz vor Ort gegeben wäre. Insofern könnten diese Maßnahmen als avantgardistische Vorhaben eingestuft werden, die neben dem Staat / der Verwaltung ebenso die Zivilgesellschaft integrieren. Obwohl der systemische Charakter sichtbar wird, sollte von Fall zu Fall geprüft werden in wieweit der Grad einer regionalen Verantwortung seitens von Unternehmen wirklich vorliegt. Erst dann ist eine Einordnung in das Reifegradmodell sinnvoll. Einen ersten Orientierungspunkt bietet eine regionale Datenbank seitens der Bertelsmann-Stiftung, die solche Maßnahmen auf einer Platform bündelt $(<$ https://regional-engagiert.de $>)$.

Die hier aufgezeigte Einordnung ist ein erster Schritt und verdeutlicht auch die Schwierigkeiten einer Systematisierung von CSpR-Maßnahmen und deren Zuordnung zur Ortsführung. Sie dient in diesem Stadium als erste Bestandaufnahme für eine weiterführende wissenschaftliche Betrachtung und vor allem dazu, einen Diskurs und die Rolle der Ortsführung / „Place Leadership“ im Rahmen eines räumlichen Unternehmensmanagements zu eröffnen.

\subsection{Tendenzen und Handlungsempfehlungen}

Wie ist das Phänomen des räumlichen Unternehmensengagements bzw. oder sogar eines räumlichen Unternehmensengagements mit einer Ortsführungsfunktion „Place Leadership" nun einzuschätzen? Es handelt sich bei diesen Phänomenen bislang um keine flächendeckende Erscheinung, weder in Agglomerationen noch in Kleinstädten. Die gezeigten Beispiele entfalten eher eine Pionierwirkung und gehen oft auf besonders proaktive Familienunternehmen oder Hidden Champions, zurück, die teils mit Hilfe von öffentlichen Mitteln oder Stiftungskapital umgesetzt werden (vgl. auch GrafFENBERGER \& GÖRMAR 2020). Ferner lassen sich die Beispiele nicht verallgemeinern, sondern sind Speerspitzen eines neuen Trends, der sowohl in der Förderpraxis und Forschung wachsende Beachtung erfährt. Überwiegend handelt es sich aber nach wie vor um CSpR 0.0 und 1.0 Maßnahmen, oder gar um CSR-Vorhaben ohne eine explizit räumliche Komponente. Was ist für eine bessere Wirkung im Stadtentwicklungskontext nötig? Hier können erste Anknüpfungspunkte an bereits regional erfolgreiche Vorhaben unterstützend wirken, etwa aus dem Bereich der Wirtschaftsförderung, die vielerorts oftmals unter Beteiligung von Unternehmen aktiv sind (ZwICKER-SCHWARM 2013). Dabei ist die Teilhabe von Unternehmen in diesem Bereich naheliegend, da hier auf Erfahrungseffekte zurückgegriffen werden kann, die einen Mehrwert für diese spezifische Aufgabe generieren. Gerade im ländlichen Raum gilt es Kompetenzen und Erfahrungen zu bündeln. Beispiele zur Regionalentwicklung durch lokales Engagement im unternehmerischen Verbund haben sich aus dem oben genannten Projekt „Unternehmen für die Region“" entwickelt (z.B. die Verantwortungspartner für Lippe des Gewerbe- und 
Innovationszentrums Lippe-Detmold GILDE GmbH, vgl. MerCHEL 2017). Mancherorts sind bereits kommunale Ansprechpartner und/ oder Vermittler vorhanden, die das Unternehmensengagement bedarfsorientiert auf Projekte abstimmen und auch Organisationsstrukturen anbieten bzw. auf unternehmensbasiertes Place Leadership vorbereitet sind (z.B. Stadtteilpatenschaften, vgl. Albers \& Hartenstein 2017a). Ebenso können kommunale Unternehmen gerade in Kleinstädten eine Vorreiterrolle spielen - etwa öffentliche oder gemeinnützige Wohnungsunternehmen, die bereits als Quartiersentwickler aktiv sind (KitZMAnN 2018). Auch wenn der Mehrwert des räumlich unternehmerischen Engagements nicht immer auf den ersten Blick erkennbar ist, genießen die vorgestellten Projekte, Maßnahmen und Initiativen oft eine breite Anerkennung und Zustimmung seitens der Bevölkerung - gerade weil sie die (sozial-)räumliche Dimension beachten und auch auf Bürgerbeteiligungsformate gesetzt wird. Dabei sind eben Pioniere, Vermittler und Grenzgänger zwischen den Sektoren notwendig. Denn die Interessenslagen und Gestaltungsvorstellungen können sehr divers sein. Beispiele für ambitioniertes aber am öffentlichen Bedarf vorbei geplantes Unternehmensengagement gibt es viele, oft mit hohen Folgekosten für die öffentliche Hand. Wichtig ist deshalb eine hinreichende Bedarfsfeststellung, die auch in der Verwaltung durch eine entsprechende Regiestelle angesiedelt sein kann. Klar ist auch, dass die Gestaltungsspielräume zwischen Unternehmen und den Kommunen den Rahmen hoheitlicher Aufgabenfelder weder unterwandern noch eine gemeinwohlorientierte Stadtplanung in Frage stellen dürfen. Unabhängig davon würde eine systematische Erhebung solcher CSpR-Initiativen in Deutschland sicher Licht ins Dunkel bringen und sowohl für Forschung als auch Praxis von Mehrwert sein. Potential (für mehr thematische Aufmerksamkeit) bietet auch eine kontinuierliche Anknüpfung an nahestehende Forschungsbereiche - Welche Rolle spielen CSpR und Place Leadership etwa beim Thema „Gleichwertige Lebensverhältnisse"

Die Sammlung von Best-Practice-Beispielen ist der Anfang für eine weitere zielgerichtete Erforschung von unterschiedlichen Typen für solch überinstitutionellen Strukturen und ihre räumlichen Wirkungen. Die hier dargelegte Verbindung zum Place Leadership Konzept erweitert die Betrachtung über die konzeptionelle und wirkungsorientierte Ebene hinaus in Richtung Entscheidungs- und Führungsebene. Nur so kann Wissen auf Seiten der beteiligten Akteure über die Konzeption und Durchführung von CSpR-Projekten akkumuliert werden. Denn gerade in Kleinstädten kann ein systematisches Engagement oder gar eine Ortsführung eine breite Wirkung entfalten.

\subsection{Fazit}

CSpR-Projekte können sowohl für Unternehmen als auch die Städte und Regionen nützlich sein - etwa durch verbesserte Standortfaktoren, erhöhte Mitarbeitermotivation oder größere Standortakzeptanz für die Unternehmen und eine Revitalisierung, zusätzliche Impulse oder Flächeninwertsetzungsprozesse für die Stadt. Unternehmen leisten 
mit ihrem Engagement ferner oft einen entscheidenden Beitrag für die Aufrechterhaltung regionaler Daseinsvorsorge und sind starke Identitätsanker. Regionales Unternehmensengagement kann allerdings auch einseitige Stadtstrukturen fördern, urbane Diversität verhindern oder sogar zerstören - sichtbar an Standorten, die durch dominante Unternehmen oder Branchen geprägt sind oder lange Zeit waren. Vor diesem ambivalenten Hintergrund ist auch das Zusammendenken von regionalem Unternehmensengagement und Ortsführung zu sehen. Gleichzeitig sind viele Maßnahmen, trotz einer grundsätzlichen Bereitschaft insbesondere bei KMU und Familienbetrieben, noch unkoordiniert und wenig zielgerichtet - eine Feststellung, auf die auch die angesprochene Studie der Bertelsmann-Stiftung hinweist (LABIGNE et al. 2018). CSpR-Projekte sind überwiegend in Städten mit starker Unternehmenslandschaft zu finden, wirtschaftlich schwache Kleinstädte profitieren deshalb kaum und bleiben infrastrukturell unterentwickelt. Gerade große, überregional agierende Unternehmen und besonders Energie-, Mobilitäts- oder Kommunikationsversorger könnten hier Verantwortung übernehmen (KITZMANN 2018). Unternehmen stellen einen Bestandteil komplexer Stadtgesellschaften dar. Sie müssen daher lernen, gemeinschaftlich mit Bürgern und Kommunen zu wirken. Kooperationen, partnerschaftliche Projekte und abgestimmte Engagementstrategien sind deshalb ein vielversprechender Weg. Hier gilt es, bestehende Hindernisse, Kommunikationsund Verständnishürden abzubauen und geeignete überinstitutionelle Plattformen für Vernetzung auszubauen.

\section{Bibliographie}

Albers H.-H. (2011), Corporate Urban Responsibility. Frankfurt, Campus.

Albers H.-H. \& Hartenstein F. (eds.) (2017a), CSR und Stadtentwicklung: Unternehmen als Partner für eine nachhaltige Stadtentwicklung. Berlin, Springer.

Albers H.-H. \& Hartenstein, F. (2017b), Silicon Valley Urbanism: Die Stadt vom Internet aus neu denken? In: StadtBauwelt, 26/2017, pp. 17-25.

Albers H.-H. \& Suwala L. (2018), Unternehmensengagement als Standortfaktor. Mit der Wirtschaft attraktive (Klein-)Städte schaffen. In: Informationen zur Raumentwicklung, 6, pp. 50-57.

Albers H.-H. \& Suwala L. (2020a), Family firms and corporate spatial responsibilities in Germany - implication on urban and regional planning and management. In: BASCo R., Stough R., Suwala L. (eds.), Family Business and Regional Development. London, Routledge.

Albers H.-H. \& Suwala L. (2020b), Place Leadership and Corporate Spatial Responsibilities. In: Sotarauta M., Beer A. (eds.), Handbook on City and Regional Leadership. Cheltenham, Edward Elgar Publishing.

ANDERTON D. (2017), Changing leadership in peripheral city region development: The case of Liverpool's high technology sectors, In: Local Economy, 32(4), pp. 352-373. 
BÄUmer K., LANGguth F. \& SChilcher C. (2019), Unternehmensengagement am heimischen Standort - wie Synergien zwischen Unternehmen und Region erschlossen werden können. In: HüLz M., KüHNE O., WebER F. (eds), Heimat. RaumFragen: Stadt - Region - Landschaft. Wiesbaden, Springer VS, pp. 215-230.

Basco R. \& Suwala L. (2020), Spatial Familiness - A bridge between family business and economic geography. In: CALABRò, A. (ed.): A research agenda for family business. A way ahead for the field. Northampton (MA), Edward Elgar Cheltenham.

Basco R., Stough R., Suwala L. (eds.) (2020), Family Business and Regional Development. London, Routledge.

BBSR (ed.) (2016), Zukunft Kleinstadt - Potenziale von Kleinstädten in peripheren Lagen. BBSR-Sonderpublikation.

Beer, A. \& Clower T. (2014), Mobilising leadership in cities and regions. In: Regional Studies, Regional Science, 1(1), pp. 5-20.

Bellandi M., Plechero M. \& Santini E. (2018), Oligarchic place leadership and resistance to change in industrial districts. Working Paper N. 18/2018. DISEI - Università degli Studi di Firenze.

Beschorner T. (2010), Corporate Social Responsibility und Corporate Citizenship: Theoretische Perspektiven für eine aktive Rolle von Unternehmen. In: BACKHAUS-MAUl H., BIEDERMANN C., Nährlich S., Polterauer J. (eds.), Corporate Citizenship in Deutschland. VS Verlag, pp.115-130.

Brinkerhoff D.W. \& Brinkerhoff J.M. (2011), Public-private partnerships: Perspectives on purposes, publicness, and good governance. In: Public Administration and Development, 31(1), pp. 2-14.

Brinkhoff S., Suwala L. \& Kulke E. (2015), Managing innovation in 'localities of learning' in Berlin and Seville. In: MiceK G. (ed.), Understanding Innovation in Emerging Economic Spaces. Farnham, Ashgate, pp. 11-31.

BürChER S. \& MAYER H. (2018), Are there differences in social capital related to corporate regional engagement in dynamic and less dynamic non-core regions? In: European Planning Studies, 26(2), pp. 342-364.

Brandt M., Dahlbeck E., Flögel F., Gärtner S., Schlieter D. \& Schilcher C. (2019), Raum und Unternehmen: Zur Funktionsweise von Unternehmensengagement in Regionen mit Entwicklungsbedarf. Baden-Baden, Nomos.

Coca-Stefaniak J.A., Parker C., Quin S., Rinaldi R. \& Byrom J. (2009), Town centre management models: A European perspective. In: Cities, 26(2), pp. 74-80.

Collinge C. \& Gibney J. (2010), Connecting place, policy and leadership. In: Policy Studies, 31(4), pp. 379-391.

De Ruiter M., Schaveling J., Ciulla J.B., \& Nijhof A. (2018), Leadership and the Creation of Corporate Social Responsibility: An Introduction to the Special Issue. In: Journal of Business Ethics, 151, pp. 871-874.

Devinney T. M. (2011), Social responsibility, global strategy, and the multinational enterprise: Global monitory democracy and the meaning of place and space. In: Global Strategy Journal, 1(3-4), pp. 329-344.

Enright M., Hunziker R., Mendle R., Reemes J. \& Vancutsem D. (2016), How cities and business can work together for growth. Global Cities Business Alliance. Discussion Paper June. $\quad<\mathrm{https} / /$ www.pwc.com/gx/en/psrc/pdf/gcba-cities-business-growth.pdf> (letzter Zugriff: 21 November 2019). 
Ezkowitz H. \& LeYdesdorfF L. (2000), The dynamics of innovation: from National Systems and "Mode 2" to a Triple Helix of university-industry-government relations, In: Research Policy, 29(2), pp. 109-123.

GAILING L. \& IBERT O. (2016), Schlüsselfiguren: Raum als Gegenstand und Ressource des Wandels. In: Raumforschung und Raumordnung, 74(5), pp. 391-403.

GARnER J. (ed.) (1992), The Company town: architecture and society in the early industrial age. Oxford. Oxford University Press.

Gibney J., Copeland S. \& Murie A. (2009), Toward a 'new' strategic leadership of place for the knowledge-based economy, In: Leadership, 5(1), pp. 5-23.

GrafFenberger M. \& Görmar F. (2020), Family Firms and Corporate Responsibility in Peripheral Regions. In: Basco R., Stough R., Suwala L. (eds.), Family Business and Regional Development. London, Routledge.

Hambleton R. (2011), Place-based leadership in a global era. In: Commonwealth Journal of Local Governance, 8/9 (May-November), pp. 8-32.

HAmbleton R. (2015), Leading the inclusive city: Place-based innovation for a bounded planet. Bristol, Policy Press.

Hanson R., Wolman H., Connolly D., Pearson K. \& McManmon R. (2010), Corporate citizenship and urban problem solving: The changing civic role of business leaders in American cities. In: Journal of Urban Affairs, 32(1), pp. 1-23.

HARRISON J. (2014), The rise of the non-state 'place-based' economic development strategy, In: Local Economy, 29(4-5), pp. 453-468.

Hiss S. (2006), Warum übernehmen Unternehmen gesellschaftliche Verantwortung? Ein soziologischer Erklärungsversuch, Frankfurt, Campus.

Horlings I. \& PADT F. (2013), Leadership for sustainable regional development in rural areas: Bridging personal and institutional aspects, In: Sustainable Development, 21(6), pp. 413-424.

HoyT L.M. (2003), The business improvement district: An internationally diffused approach to revitalization. Cambridge (MA), MIT.

Hu X. \& Hassink R. (2017), Place leadership with Chinese characteristics? A case study of the Zaozhuang coal-mining region in transition. In: Regional Studies, 51(2), pp. 224-234.

Idris-Soven A., IDris-Soven E. \& Vaughan M.K. (1978), The world as a company town: Multinational corporations and the social sciences. The Hague, Mouton.

Judd D. \& PARKInson M. (1990), Leadership and Urban Regeneration: Cities in North America and Europe. London, Sage.

KaYden J. (2000), Privately owned public space: the New York City experience. New York, John Wiley \& Sons.

Kiese M. \& SCHIEK M. (2016), Unternehmerische Verantwortung in der Stadt- und Regionalentwicklung. Standort, 40(1), pp. 9-12.

KITZMANN R. (2018), Unternehmerische Quartiersentwicklung - Gründe für und wider wohnungswirtschaftliches Engagement. In: Informationen zur Raumentwicklung, 5, pp. 80-91.

Kitzmann R. \& Suwala L. (2018), Oberschöneweide oder Oberschweineöde? Ein Berliner Altindustriestandort im Zeichen der Revitalisierung. In: Geographische Rundschau, 70(11), pp. 42-48.

Knieling J., Othengrafen F. \& Preising T. (2012), Privatisierung von Stadt- und Regionalentwicklung: Gesellschaftlicher Nutzen oder Verwirklichung von Unternehmenszielen? 
„Corporate Spatial Responsibility“ oder „Corporate Spatial Strategy“?, In: Raumforschung und Raumordnung, 70 (5), pp. 451-464.

Labigne A., Gilroy P., Kononykhina O., Hollmann D., Schilcher C. \& Riess R. (2018), CC-Survey: Unternehmensengagement und Corporate Citizenship in Deutschland - bessere Daten für besseres Unternehmensengagement. Studie der Bertelsmann-Stiftung.

Labigne A., Gilroy P., Kononykhina O., Hollmann D. \& Schilcher C. (2019), Unternehmen Gestalten Regionen: Vier Herausforderungen vor Ort. Essen.

Lang T., Görmar F., Graffenberger M., Vonnahme L. \& Porsche L. (2019), Hidden Champions und Stadtentwicklung: die wirtschaftliche und gesellschaftliche Bedeutung innovativer Unternehmen für Kleinstädte in peripherer Lage. Bonn, BBSR.

Lange D., Rohn K., Piesbergen M., Schmitz H. \& Suwala L. (2011), Nachhaltige Vitalisierung des kreativen Quartiers um den Campus Berlin Charlottenburg. Berlin, WISTA Management.

Lengauer L. \& TöDtLing F. (2010), Regional embeddedness and corporate regional engagement: evidence from three industries in the Austrian region of Styria. Paper Presented at the $8^{\text {th }}$ European Urban \& Regional Studies Conference. Vienna, September,15-17.

Liddle J., Potluka O., Quinn M., Anderton D. \& Bartling H. (2017), Editorial, In: Local Economy, 32 (4), pp. 267-272.

Merchel R. (2017), Verantwortungspartner für Lippe - ein Beitrag zur Regionalentwicklung durch lokales Engagement im unternehmerischen Verbund. In: Albers H.-H. \& HarTeNSTEIN F. (eds.), CSR und Stadtentwicklung. Berlin. Springer Gabler, pp. 371-387.

Mozingo L.A. (2011), Pastoral Capitalism: A History of Suburban Corporate Landscapes, Cambridge (MA), MIT Press.

Nicholds A., Gibney J., Mabey C. \& HaRt D. (2016), Making sense of variety in place leadership: The case of England's smart cities. In: Regional Studies, 51(2), pp. 249-259.

Plaza B. (2008), On some challenges and conditions for the Guggenheim Museum Bilbao to be an effective economic re-activator. In: International Journal of Urban and Regional Research, 32(2), pp. 506-517.

Potluka O., Kalman J., Musialkowska I. \& Idczak P. (2017), Non-profit leadership at local level: Reflections from Central and Eastern Europe, In: Local Economy, 32(4), pp. 297-315.

RAaGmaA G. \& KeERBer A (2017), Regional higher education institutions in regional leadership and development. In: Regional Studies, 51(2), pp. 260-272.

Rossiter W. \& Sмiтh D. J. (2017), Institutions, place leadership and public entrepreneurship: Reinterpreting the economic development of Nottingham. In: Local Economy, 32(4), pp. 374-392.

SCHIEK M. (2017), Corporate Regional Responsibility (CRR). Lemgo, Rohn.

SCHNEIDER A. (2012), Reifegradmodell CSR - eine Begriffserklärung und Abgrenzung. In: SchneIder A., SchmidPeter R. (eds.), Corporate Social Responsibility, Verantwortungsvolle Unternehmensführung in Theorie und Praxis. Berlin, Springer Gabler, pp. 17-38.

Simon H. (1996), Die heimlichen Gewinner: Die Erfolgsstrategien unbekannter Weltmarktführer (Hidden Champions). Frankfurt a.M., Campus.

Sotarauta M. (2005), Shared Leadership and Dynamic Capabilities in Regional Development. In: Halkier S. (ed.), Regionalism Contested: Institution, Society and Governance. Cornwall, Ashgate, pp. 53-72. 
Sotarauta M. (2016), Place leadership, governance and power. In: Administration 64(3-4), pp. 45-58.

Sotarauta M., Beer A., \& Gibney J. (2017), Making sense of leadership in urban and regional development. In: Regional Studies, 51(2), pp. 187-193.

Sotarauta M., Horlings I. \& Liddle J. (eds.) (2012), Leadership and change in sustainable regional development. Abingdon, Routledge.

Stimson R.J., Stough R.R. \& Roberts B.H. (2002), Regional Economic Development: Analysis and Planning Strategy. Berlin, Springer.

Stimson R., Stough R.R. \& Salazar M. (2009), Leadership and Institutions in Regional Endogenous Development. Northampton, Edward Elgar.

Stough R.R. (2003), Strategic management of places and policy, In: Annals of Regional Science, 37(2), pp. 179-201.

Suwala L. (2014), Kreativität, Kultur und Raum: ein wirtschaftsgeographischer Beitrag am Beispiel des kulturellen Kreativitätsprozesses. Berlin, Springer-Verlag.

Suwala L. (2015), Cultural districts. In: Wherry F., Schor J. (eds.), The Sage Encyclopedia of Economics and Society. Thousand Oaks, Sage CA, pp. 511-513.

Suwala L. (2019), Management Geography - Standortentscheidungen, Netzwerke und Raumbilder nordamerikanischer und japanischer Familienunternehmen.

Suwala L. \& Dannenberg P. (2009), Cluster- und Innovationspolitik maßgeschneidert - Das Beispiel Adlershof in Berlin. In: Standort, 33(4), pp. 104-112.

Suwala L. \& Micek G. (2018), Beyond clusters? Field configuration and regional platforming: The Aviation Valley initiative in the Polish Podkarpackie region. In: Cambridge Journal of Regions, Economy and Society, 11(2), pp. 353-372.

Suwala L., Kulke E. \& Gade K. (2018), Zentralamerika (Nicaragua, Honduras, Guatemala, Belize) Bericht zur Hauptexkursion 2017. Arbeitsberichte Geographisches Institut Heft 195. Berlin.

Sydow J., Lerch F., Huxham C. \& HibBert P. (2011), A silent cry for leadership: Organizing for leading (in) clusters. In: The Leadership Quarterly, 22(2), pp. 328-343.

VAN VELSOR E. (2009), Introduction: Leadership and corporate social responsibility. In: Corporate Governance, 9(1), pp. 3-6.

Voegtlin C., Patzer M. \& Scherer A. (2012), Responsible leadership in global business: A new approach to leadership and its multi-level outcomes. In: Journal of Business Ethics, 105(1), pp. 1-16.

Vonnahme L., Graffenberger M., Görmar F. \& Lang T. (2018), Kaum beachtet, gemeinsam stark: versteckte Potenziale von Kleinstädten mit Hidden Champions. In: Informationen zur Raumentwicklung 6, pp 38-49.

Waldman A.D., Siegel D. (2008), Defining a socially responsible leader. In: The Leadership Quarterly, 19(1), pp. 117-131.

Zwicker-SchWARm D. (2013), Kommunale Wirtschaftsförderung 2012: Strukturen, Handlungsfelder, Perspektiven. Difu. 